\title{
Catechin complexed with lysine has potent antitumor activities in human breast cancer xenograft model
}

\author{
Elias N Katsoulieris and Amanda Canas Rodriguez
}

University of Louvain (UCL) Medical School, Brussels, Belgium

bioRxiv has withdrawn this preprint as it was posted without proper authorization therefore violating confidentiality agreements between involved parties. 